\title{
EMILIE ROUVRE
}

\section{GÉRARD GAGNEUX}

\section{Formulation forte entropique de lois scalaires hyperboliques-paraboliques dégénérées}

Annales de la faculté des sciences de Toulouse $\sigma^{e}$ série, tome 10 , $\mathrm{n}^{\mathrm{o}} 1$ (2001), p. 163-183

<http://www.numdam.org/item?id=AFST_2001_6_10_1_163_0>

(C) Université Paul Sabatier, 2001, tous droits réservés.

L'accès aux archives de la revue «Annales de la faculté des sciences de Toulouse » (http://picard.ups-tlse.fr/ annales/) implique l'accord avec les conditions générales d'utilisation (http://www.numdam.org/conditions). Toute utilisation commerciale ou impression systématique est constitutive d'une infraction pénale. Toute copie ou impression de ce fichier doit contenir la présente mention de copyright.

\section{NumDam}

Article numérisé dans le cadre du programme Numérisation de documents anciens mathématiques http://www.numdam.org/ 


\title{
Formulation forte entropique de lois scalaires hyperboliques-paraboliques dégénérées ${ }^{(*)}$
}

\author{
Emilie Rouvre ET GÉRARd GaGNeUX ${ }^{(1)}$
}

\begin{abstract}
Résumé. - On établit l'existence, l'unicité et la dépendance continue par rapport à la donnée initiale des solutions "fortes" entropiques pour des problèmes de Cauchy-Dirichlet associés à la loi scalaire: $u_{t}-\Delta \varphi(u)-$ $\operatorname{div}(\nu(u) \mathbf{G})=0$. On se place ici dans le cas où $\varphi$ est une fonction croissante au sens large et constante sur un ensemble $\mathcal{L}^{1}$ - non négligeable: la loi scalaire considérée introduit donc une équation hyperbolique-parabolique dégénérée, mêlant des phénomènes de parabolicité et d'hyperbolicité non linéaires.
\end{abstract}

\begin{abstract}
We consider a class of parabolic-hyperbolic degenerate equations $u_{t}-\Delta \varphi(u)-\operatorname{div}(\nu(u) \mathbf{G})=0$ in $\left.Q=\right] 0, T[\times \Omega$, associated to Cauchy-Dirichlet problem. The special feature of our framework is double: 1) the nonlinear equation degenerates into first order hyperbolic type if the unknown value $u$ is less than a critical value, i.e., really on an interval of solution values; 2) we are concerned with the existence, stability and uniqueness of strong entropy solutions under appropriate assumptions on the data (all the weak first partial derivatives of $u$ are bounded Radon measures and those of $\varphi(u)$ belong to $L^{2}(Q)$ ). In the spatially onedimensional case, entropy boundary conditions and jump conditions are explained taking into account simultaneously diffusion effects and convection movements to illustrate the well-posedness of the formulation.
\end{abstract}

(*) Reçu le 27 janvier 1999, accepté le 8 juin 2001

(1) Laboratoire de mathématiques appliquées à l'Industrie, Université de Pau et des Pays de l'Adour, I.P.R.A., Avenue de l'université, 64000 Pau, France. email: Emilie.Rouvre@univ-pau.fr, Gerard.Gagneux@univ-pau.fr 


\section{Introduction}

Le propos de cet article est de définir une formulation forte dans un cadre fonctionnel approprié pour bien poser au sens de Hadamard le problème aux limites $(\mathcal{P})$ suivant, d'inconnue $u$ et de type divergentiel :

$$
(\mathcal{P})\left\{\begin{array}{l}
\left.\frac{\partial u}{\partial t}-\Delta \varphi(u)-\operatorname{div}(\nu(u) \mathbf{G})=0 \text { dans } Q=\right] 0, T[\times \Omega \\
\varphi(u)=0 \text { sur } \Sigma=] 0, T[\times \partial \Omega \\
(\text { avec, le cas échéant, une condition sur la trace } \\
\text { de } \nu(u) \text { le long d'une partie ad hoc de } \Sigma) \\
u(0, .)=u_{0} \text { dans } \Omega
\end{array}\right.
$$

lorsque $\Omega$ est un ouvert borné connexe de $\mathbb{R}^{n}$ et $\varphi$ une fonction numérique, nulle sur tout l'intervalle $\left[0, \varphi_{c}\right], \varphi_{c}>0$, où l'inconnue $u$ prend a priori certaines de ses valeurs. On prend le parti de rechercher une solution telle que, lorsque $\varphi$ est identiquement nulle ou lorsque, par application d'un principe du maximum, l'inconnue $u$ prend toutes ses valeurs dans l'intervalle $\left[0, \varphi_{c}\right]$, on retrouve les résultats classiques de la formulation forte de C. Bardos, A.Y. Leroux, J.C. Nédélec [1], par opposition à la formulation affaiblie énoncée par F. Otto [16] : ainsi, pour le traitement du terme de transport, on recherchera des solutions $u$ dans $L^{\infty}(Q) \cap \overline{B V}(Q)$ et, en tirant profit de l'existence d'un terme régularisant de diffusion, on obtiendra que $\varphi(u)$ soit élément de $H^{1}(Q)$, pour un état initial suffisamment régulier.

Ce problème tient son originalité du fait que $\varphi$, fonction de classe $\mathcal{C}^{1}$, est nulle sur tout l'intervalle $\left[0, \varphi_{c}\right]$, et non en des valeurs isolées. Ici le trait essentiel de l'étude est que $\mathcal{L}^{1}\left\{\mathrm{r} \in \mathbb{R}, \varphi^{\prime}(r)=0\right\} \neq 0$ (i.e. on se place précisément hors du cadre retenu par Ph. Bénilan et R. Gariepy [3] pour établir l'existence de solutions fortes dans $L^{1}$ ) ; la fonction $\varphi$ n'est pas injective, ce qui rend inopérantes les méthodes usuelles de la diffusionconvection en milieu poreux (cf. [3], [9], [10], [20]). Ainsi, $u$ est une fonction définie sur $] 0, T\left[\times \Omega\right.$, qui en deçà de la valeur critique $\varphi_{c}$ est solution d'un problème hyperbolique non linéaire du premier ordre et, au delà, solution d'un problème parabolique dégénéré, introduisant des frontières libres. C'est pourquoi on ne pourra trouver de solutions continues, ni même $\mathcal{L}^{n+1}$-approximativement continues et on s'intéressera à l'espace des fonctions mesurables et bornées à variation bornée, dans le but d'obtenir, en vue des applications, des solutions assez fortes, au sens où les gradients des grandeurs considérées (par rapport à $t$ et à $\mathrm{x}$ ) sont des mesures de Radon sommables sur Q. En outre, on s'attache dans ce contexte à donner un sens à des expressions utiles à l'expérimentateur telles que $\frac{\partial \varphi(u)}{\partial n}$ pour définir le flux pariétal. 
D'autres approches analytiques du problème $(\mathcal{P})$ peuvent être trouvées dans les travaux récents de J. Carrillo ([6], détaillés dans [7]) qui définissent une notion de solution faible entropique, unique en son genre, ou dans les travaux de $\mathrm{Ph}$. Bénilan et $\mathrm{H}$. Touré [4] via un concept de "bonne solution" ("mild solution" en anglais), ou dans l'étude de M. Maliki et $\mathrm{H}$. Touré [14] qui introduisent une notion de "solution locale généralisée".

L'étude d'un tel problème, changeant de type sur des frontières libres, est motivée par l'analyse mathématique de diverses modélisations : citons le cas

- du trafic autoroutier (lorsque, par un phénomène d'anticipation intervenant à partir d'un seuil critique de densité, les conducteurs corrigent leur vitesse en fonction des variations de la densité locale de véhicules ( $c f$. [19] vol. I, pp. 12-13))

- des phénomènes d'écoulement en milieux poreux (dans le cas où les fonctions de perméabilités relatives restent nulles sur un intervalle $\left[0, \varphi_{c}\right]$, pour une certaine valeur $\varphi_{c}$ de la saturation des composants, dite résiduelle $(c f .[10])$ ), ou des modèles de floculation ou d'hydrosédimentation (voir sur ce point les travaux de R. Bürger et W.L. Wendland [5]).

Le plan de travail est le suivant :

En premier lieu, on établit l'existence d'une solution entropique dans le cas général où $\Omega$ est un domaine borné, lipschitzien de $\mathbb{R}^{n}$. Dans une seconde partie, en adaptant la méthode de J. Carrillo [6], on montre un résultat de comparaison, de portée très générale, dans l'esprit de l'approche des problèmes hyperboliques non linéaires du premier ordre de S.N. Kruzhkov.

Enfin, la dernière partie est consacrée à l'étude de l'unicité de la solution entropique lorsque est $\Omega=] 0,1$ [, i.e. dans le cas de mouvements unidirectionnels. Le cas monodimensionnel apporte des informations sur les conditions de bord et de choc, permettant de donner une interprétation rigoureuse de la condition de bord, justifiant ainsi la notion de solution forte entropique.

\section{Les données du problème}

Soient $\Omega$ un domaine borné et lipschitzien de $\mathbb{R}^{n}$ et $\Gamma$ sa frontière, de vecteur normal unitaire extérieur $\mathbf{n}$ défini $\mathcal{H}^{n-1}$-presque partout.

On considère le problème hyperbolique-parabolique dégénéré : chercher $u$ a priori dans $L^{\infty}(Q), 0 \leqslant u \leqslant 1 \quad \mathcal{L}^{n+1}-p$.p. dans $Q$, solution 
du problème informel :

$$
(\mathcal{P})\left\{\begin{array}{l}
\left.\frac{\partial u}{\partial t}-\Delta \varphi(u)-\operatorname{div}(\nu(u) \mathbf{G})=0 \text { dans } Q=\right] 0, T[\times \Omega \\
\varphi(u)=0 \text { sur } \Sigma=] 0, T[\times \partial \Omega \\
\text { (sous réserve d'une condition additionnelle affectant } \\
\text { la trace de } \nu(u) \text { sur une partie appropriée de } \Sigma, \\
\text { lorsque la condition précédente est de fait sans objet) } \\
u(0, .)=u_{0} \text { dans } \Omega
\end{array}\right.
$$

où :

$\nu$ est une fonction lipschitzienne sur [0,1], croissante (au sens large), vérifiant par normalisation $\nu(0)=0$ et $\varphi$ une fonction de classe $\mathcal{C}^{1}$ sur $[0,1]$, nulle sur $\left[0, \varphi_{c}\right]\left(\varphi_{c}\right.$ étant une constante telle que $\left.0<\varphi_{c}<1\right)$, strictement croissante sur l'intervalle $\left[\varphi_{c}, 1\right]$.

G désigne un vecteur fixe de $\mathbb{R}^{n}$ (ou plus généralement un champ de vecteurs sur $\bar{\Omega}$, stationnaire et lipschitzien, à divergence nulle, avec pour cas classique de référence $\mathbf{G}=\nabla P, P \in W^{2,+\infty}(\Omega)$, tel que $\left.\Delta P=0\right)$.

Enfin, on suppose que la donnée $u_{0}$ est un élément de $L^{\infty}(\Omega) \cap \overline{B V}(\Omega)$ telle que :

$\left(\mathcal{H}_{1}\right) \quad 0 \leqslant u_{0} \leqslant 1 \quad \mathcal{L}^{n}-p . p$. dans $\Omega, \varphi\left(u_{0}\right) \in H_{0}^{1}(\Omega)$ et $\Delta \varphi\left(u_{0}\right) \in \mathcal{M}_{b}(\Omega)$,

hypothèse usuelle pour obtenir dans le cas non fortement dégénéré des solutions fortes (P.Urruty [20]). De plus, cette hypothèse assure (à l'aide de la proposition 9, p.580 de [8] et sous réserve que $\Gamma$ est de classe $W^{2,+\infty}$ ) que $\frac{\partial \varphi\left(u_{0}\right)}{\partial n} \in L^{1}(\Gamma)$, donnant ainsi un sens au flux pariétal initial.

Les différentes notations utiles à la suite de notre étude sont réunies dans :

\section{Notations.}

- Soit $\mu$ une mesure de Radon sommable sur $Q$ (i.e. $\mu \in \mathcal{M}_{b}(Q)$ ) ; on désigne par $|\mu|_{\mathcal{M}_{b}(Q)}$ sa variation totale sur $Q$.

- Pour tout élément $s$ de $\mathbb{R}$, on pose :

$$
\operatorname{sign}_{0}(s)=\left\{\begin{array}{l}
-1 \text { si } s<0 \\
0 \text { si } s=0 \\
1 \text { si } s>0
\end{array}\right.
$$


et on introduit l'approximation Yosida croissante, nulle en 0 et lipschitzienne :

$$
\operatorname{sign}_{\varepsilon}(s)=\left\{\begin{array}{l}
-1 \text { si } s<-\varepsilon \\
\frac{s}{\varepsilon} \text { si }|s| \leqslant \varepsilon \\
1 \text { si } s>\varepsilon>0 .
\end{array}\right.
$$

\section{Existence d'une solution entropique}

L'élaboration d'une formulation entropique à partir d'une méthode classique de viscosité évanescente impose de définir préalablement la dérivée normale le long de $\Sigma$ pour une fonction $U$ élément de $H^{1}(Q)$, telle que $\Delta U \in \mathcal{M}_{b}(Q)$. On peut trouver une démarche similaire dans l'étude de $\mathrm{E}$. Magenes [13] où il est question de donner un sens, dans une acception affaiblie, à la notion de flux pariétal pour le problème de Stefan ( $c f$. aussi sur ce point [8], définition 3, p. 583).

DÉFInITION 1. - Soit $U \in H^{1}(Q)$ telle que $\Delta U \in \mathcal{M}_{b}(Q)$. On définit, en un sens faible, la dérivée normale $\frac{\partial U}{\partial n}$ le long de $\Sigma$ par la formule de Green généralisée :

pour tout $\Phi \in \mathcal{C}^{0}(\bar{Q}) \cap L^{2}\left(0, T ; H^{1}(\Omega)\right)$,

$$
\left\langle\left\langle\frac{\partial U}{\partial n}, \Phi_{\mid \Sigma}\right\rangle\right\rangle=\int_{Q} \nabla U \cdot \nabla \Phi d x d t+\int_{Q} \Phi d[\Delta U] .
$$

Cette expression ne dépend que de $\Phi_{\left.\right|_{\Sigma}}$, par le fait qu'elle est nulle pour tout $\Phi \operatorname{de} \mathcal{C}^{0}(\bar{Q}) \cap L^{2}\left(0, T ; H_{0}^{1}(\Omega)\right)$.

Lorsque $\nu$ est une fonction non linéaire (ce qui sera la situation considérée ici), le problème $(\mathcal{P})$, pour être bien posé au sens de Hadamard, doit être formulé d'une façon plus contraignante. Pour cela, on introduit la définition suivante qui résulte de la démarche classique de S.N. Kruzhkov et de l'analyse du comportement limite des solutions de viscosité artificielle :

DÉFINITION 2.- La fonction $u$ est dite solution entropique de $(\mathcal{P})$ si et seulement si :

$$
\begin{gathered}
u \in L^{\infty}(Q) \cap \overline{B V}(Q) \cap \operatorname{Lip}\left([0, T] ; L^{1}(\Omega)\right) \\
\frac{\partial u}{\partial t} \in L^{2}\left(0, T ; H^{-1}(\Omega)\right) \\
0 \leqslant u \leqslant 1 \quad \mathcal{L}^{n+1}-p . p . \text { dans } Q \\
\varphi(u) \in H^{1}(Q) \cap L^{\infty}\left(0, T ; H_{0}^{1}(\Omega)\right), \Delta \varphi(u) \in \mathcal{M}_{b}(Q), \\
u(0, .)=u_{0} \quad \mathcal{L}^{n}-p . p . \operatorname{dans} \Omega \\
-167-
\end{gathered}
$$


et vérifie les inégalités d'entropie :

$$
\begin{gathered}
\forall s \in[0,1], \quad \forall \Phi \in \mathcal{D}([0, T[\times \bar{\Omega}), \Phi \geqslant 0, \\
\int_{Q}|u-s| \Phi_{t} d x d t+\int_{\Omega}\left|u_{0}-s\right| \Phi(0, x) d x \\
-\int_{Q}|\nu(u)-\nu(s)| \mathbf{G} \cdot \nabla \Phi d x d t-\int_{Q} \nabla|\varphi(u)-\varphi(s)| . \nabla \Phi d x d t \\
-\operatorname{sign}_{0}(s) \int_{\Sigma}(\nu(u)-\nu(s)) \mathbf{G} \cdot n \Phi d \sigma d t-\operatorname{sign}_{0}(s)\left\langle\left\langle\frac{\partial \varphi(u)}{\partial n}, \Phi\right\rangle\right\rangle \geqslant 0 .
\end{gathered}
$$

L'existence d'une solution entropique sera justifiée par une méthode de viscosité artificielle, habituellement utilisée pour la régularisation de problèmes hyperboliques non linéaires du premier ordre ( $c f$. C. Bardos, A.Y. Leroux, J.C. Nédélec [1]). Pour $\varepsilon>0$, on définit $\varphi_{\varepsilon}=\varphi+\varepsilon I d_{[0,1]}$; on est ainsi ramené au cas où $\varphi_{\varepsilon}$ est une fonction strictement croissante et bilipschitzienne.

Par ailleurs, on considère une approximation régularisée appropriée $u_{0}^{\varepsilon}$ de la donnée $u_{0}$. Pour cela, on suppose que l'état initial n'est pas trop pathologique, au sens où (ce qui est toujours vrai en dimension 1) :

$$
\left(\mathcal{H}_{2}\right) \quad\left\{\begin{array}{c}
\Omega_{+}=\left\{x \in \Omega, \varphi\left(u_{0}(x)\right)>0\right\} \text { est un ouvert, } \\
\Omega_{0}=\operatorname{Int}\left(\bar{\Omega} \backslash \overline{\Omega_{+}}\right) \text {est non vide, de frontière régulière, } \\
\text { de classe } W^{2,+\infty} .
\end{array}\right.
$$

On choisit alors l'approximation suivante :

$$
u_{0}^{\varepsilon}=\chi\left(\Omega_{+}\right) \varphi_{\varepsilon}^{-1}\left(\varphi\left(u_{0}\right)\right)+\chi\left(\Omega_{0}\right) z_{\varepsilon}, \text { définie } \mathcal{L}^{n}-p . p \text {. dans } \Omega,
$$

où $z_{\varepsilon} \in H_{0}^{1}\left(\Omega_{0}\right) \cap H^{2}\left(\Omega_{0}\right)$ est la solution du problème elliptique

$$
\left\{\begin{array}{l}
z_{\varepsilon}-\varepsilon \Delta z_{\varepsilon}=u_{0} \text { dans } \Omega_{0} \\
\left(z_{\varepsilon}\right)_{\mid \partial \Omega_{0}}=0 .
\end{array}\right.
$$

Les propriétés qui résultent de cette construction sont les suivantes :

$$
\begin{aligned}
& u_{0}^{\varepsilon} \in H_{0}^{1}(\Omega) ; 0 \leqslant u_{0}^{\varepsilon} \leqslant 1 \quad \mathcal{L}^{n}-p . p . \text { dans } \Omega, 0 \leqslant z_{\varepsilon} \leqslant \varphi_{c} \quad \mathcal{L}^{n}-p . p . \text { dans } \Omega_{0} ; \\
& \varphi_{\varepsilon}\left(u_{0}^{\varepsilon}\right)=\chi\left(\Omega_{+}\right) \varphi\left(u_{0}\right)+\varepsilon \chi\left(\Omega_{0}\right) z_{\varepsilon}, \operatorname{car} \varphi\left(z_{\varepsilon}\right)=0 ; \\
& u_{0}^{\varepsilon} \longrightarrow u_{0} \text { dans } L^{p}(\Omega) \text { pour tout } p \text { fini ; }
\end{aligned}
$$

il existe une constante $C>0$, indépendante de $\varepsilon$, telle que :

$T V_{\Omega}\left(u_{0}^{\varepsilon}\right)+\left\|\varphi_{\varepsilon}\left(u_{0}^{\varepsilon}\right)\right\|_{H_{0}^{1}(\Omega)}+\left|\operatorname{div}\left(\nu\left(u_{0}^{\varepsilon}\right) \mathbf{G}\right)\right|_{\mathcal{M}_{b}(\Omega)}+\left|\Delta \varphi_{\varepsilon}\left(u_{0}^{\varepsilon}\right)\right|_{\mathcal{M}_{b}(\Omega)} \leqslant C$.

Le point de départ de l'étude est constitué du résultat classique suivant, obtenu par le théorème de point fixe de Schauder-Tychonov ( $c f .[10]$, par exemple) 
THÉORÈME 1. - Sous les hypothèses $\left(\mathcal{H}_{1}\right)$ et $\left(\mathcal{H}_{2}\right)$, pour tout $\varepsilon>0$, il existe un unique élément $u_{\varepsilon}$ dans $H^{1}(Q) \cap L^{\infty}\left(0, T ; H_{0}^{1}(\Omega)\right)$ tel que :

$$
\begin{gathered}
0 \leqslant u_{\varepsilon} \leqslant 1 \quad \mathcal{L}^{n+1}-\text { p.p. dans } Q \\
\text { et } \varphi_{\varepsilon}\left(u_{\varepsilon}\right) \in L^{\infty}\left(0, T ; H_{0}^{1}(\Omega)\right) \cap L^{2}\left(0, T ; H^{2}(\Omega)\right),
\end{gathered}
$$

solution du problème autonome, régularisé et non dégénéré :

$$
\left(\mathcal{P}_{\varepsilon}\right)\left\{\begin{array}{l}
\frac{\partial u_{\varepsilon}}{\partial t}-\Delta \varphi_{\varepsilon}\left(u_{\varepsilon}\right)-\operatorname{div}\left(\nu\left(u_{\varepsilon}\right) \mathbf{G}\right)=0 \quad \mathcal{L}^{n+1}-p . p . \text { dans } Q \\
u_{\varepsilon}(t, .)_{\mid \delta \Omega}=0 \text { p.p en } t, \mathcal{H}^{n-1}-p . p . \text { sur } \Gamma \\
u_{\varepsilon}(0, .)=u_{0}^{\varepsilon} \mathcal{L}^{n}-p . p . \text { dans } \Omega .
\end{array}\right.
$$

On établit alors les estimations a priori suivantes, dont on pourra trouver le détail du mode d'obtention dans [10] (chap. 4, pp. 160-164) :

- Il existe une constante $C_{0}$ indépendante de $\varepsilon$ telle que, $\left.\left.\forall t \in\right] 0, T\right]$,

$$
\begin{aligned}
\left|\frac{\partial u_{\varepsilon}}{\partial t}(t, .)\right|_{L^{1}(\Omega)} & \leqslant\left|\Delta \varphi_{\varepsilon}\left(u_{0}^{\varepsilon}\right)\right|_{\mathcal{M}_{b}(\Omega)}+\left|\operatorname{div}\left(\nu\left(u_{0}^{\varepsilon}\right) \mathbf{G}\right)\right|_{\mathcal{M}_{b}(\Omega)} \\
& \left.\leqslant C_{0} \quad \text { (d'après les propriétés de } u_{0}^{\varepsilon}\right)
\end{aligned}
$$

- Il existe deux constantes $C_{1}$ et $C_{2}$ indépendantes de $\varepsilon$ telles que, p.p. $t \in$ ] $0, T[$,

$$
\int_{\Omega}\left|\nabla u_{\varepsilon}(t)\right|_{1} d x \leqslant\left[T V\left(u_{0}^{\varepsilon}\right)+C_{1}\right]+C_{2} \int_{0}^{t} \int_{\Omega}\left|\nabla u_{\varepsilon}(s)\right|_{1} d x d s,
$$

où $\left|\nabla u_{\varepsilon}(t)\right|_{1}=\sum_{i=1}^{n}\left|\frac{\partial u_{\varepsilon}}{\partial x_{i}}(t,).\right|$.

On conclut par le lemme de Gronwall que $\left(u_{\varepsilon}\right)_{\varepsilon>0}$ est une suite bornée de $L^{\infty}\left(0, T ; W^{1,1}(\Omega)\right)$.

- Il existe une constante $C$ indépendante de $\varepsilon$ telle que (cf. [10] chap. 3, p. 99 à 103),

$$
\left\|\varphi\left(u_{\varepsilon}\right)\right\|_{L^{\infty}\left(0, T ; H_{0}^{1}(\Omega)\right)}+\left\|\frac{\partial \varphi\left(u_{\varepsilon}\right)}{\partial t}\right\|_{L^{2}(Q)}+\sqrt{\varepsilon}\left\|u_{\varepsilon}\right\|_{L^{\infty}\left(0, T ; H_{0}^{1}(\Omega)\right)} \leqslant C .
$$

Il s'ensuit que de la suite $\left(u_{\varepsilon}\right)_{\varepsilon>0}$, on peut extraire une sous-suite admettant dans $\mathcal{C}^{0}\left([0, T] ; L^{1}(\Omega)\right)$ un point d'accumulation $u$ qui répond aux critères de la définition 2. Ainsi peut-on énoncer le résultat d'existence : 
THÉORÈME 2. - Sous les hypothèses $\left(\mathcal{H}_{1}\right)$ et $\left(\mathcal{H}_{2}\right)$, il existe au moins une solution entropique (au sens de la définition 2) du problème $(\mathcal{P})$.

La fonction $u$ vérifie en particulier la condition locale d'entropie : pour tout $s \in[0,1]$, on a au sens des distributions $\operatorname{sur} Q$, puis, selon un résultat de L. Schwartz [18], au sens des mesures sur $Q$ :

$$
\frac{\partial|u-s|}{\partial t}-\nabla|\nu(u)-\nu(s)| . \mathbf{G} \leqslant \Delta|\varphi(u)-\varphi(s)|
$$

et $a$ fortiori, pour les choix de $s=0$ et $s=1$, l'équation de conservation

$$
\frac{\partial u}{\partial t}-\operatorname{div}(\nu(u) \mathbf{G})-\Delta \varphi(u)=0 \text { est vérifiée dans } \mathcal{M}_{b}(Q) \text { et } L^{2}\left(0, T ; H^{-1}(\Omega)\right) \text {. }
$$

En outre, on peut remarquer que toute solution entropique vérifie (pour le moment de manière informelle), la condition d'entropie sur la frontière (dite condition de bord) :

pour tout $s \in[0,1]$, et presque partout $\operatorname{sur} \Sigma$;

$$
\begin{aligned}
& \left(\operatorname{sign}_{0}(u-s)+\operatorname{sign}_{0}(s)\right)(\nu(u)-\nu(s)) \mathbf{G . n} \\
+ & {\left[\operatorname{sign}_{0}(s) \frac{\partial \varphi(u)}{\partial n}+\frac{\partial|\varphi(u)-\varphi(s)|}{\partial n}\right] \leqslant 0 . }
\end{aligned}
$$

Ceci vient essentiellement du fait que $\Delta|\varphi(u)-\varphi(s)| \in \mathcal{M}_{b}(Q)$, et donc, selon la définition 1 , on peut donner un sens à la dérivée normale $\frac{\partial|\varphi(u)-\varphi(s)|}{\partial n}$.

\section{Résultat de comparaison}

Ici, on se propose d'établir un résultat de comparaison en adaptant la méthode récemment présentée par J. Carrillo [6] et qui, par sa mise en œuvre, discrimine les régions a priori inconnues d'hyperbolicité et de parabolicité de l'équation.

Pour ce faire, on introduit les notations suivantes et on énonce divers lemmes :

Notations. - Soit $u$ une solution entropique du problème $(\mathcal{P})$; on pose

$$
\begin{aligned}
& Q_{0}^{u}=\{(t, x) \in Q, \varphi(u(t, x))=0\}=\left\{(t, x) \in Q, 0 \leqslant u(t, x) \leqslant \varphi_{c}\right\}, \\
& Q_{+}^{u}=\{(t, x) \in Q, \varphi(u(t, x))>0\}=\left\{(t, x) \in Q, \varphi_{c}<u(t, x) \leqslant 1\right\},
\end{aligned}
$$

respectivement régions d'hyperbolicité et de parabolicité, $\mathcal{L}^{n+1}$ - mesurables. 
Formulation forte entropique de lois scalaires hyperboliques-paraboliques dégénérées

Lemme 1. - Pour toute solution entropique $u$ et tout réel $s$ de $[0,1]$, tel que $\varphi(s)>0$, on $a$ :

$$
\operatorname{sign}_{0}(u-s)=\operatorname{sign}_{0}(\varphi(u)-\varphi(s)) \text { presque partout dans } Q .
$$

Lemme 2. - Pour toute solution entropique $u$ du problème $(\mathcal{P})$,

$$
\nabla \varphi(u)=0 \quad \mathcal{L}^{n+1}-\text { p.p. dans } Q_{0}^{u} .
$$

Démonstration. - Ce résultat vient du lemme de M. Marcus et V.J. Mizel (cf. [15]) qui énonce que pour $p \in[1,+\infty]$, pour $U \in W^{1, p}(\Omega), \nabla U=$ 0 presque partout sur $U^{-1}(\mathcal{N})$, où $\mathcal{N}$ est un ensemble $\mathcal{L}^{1}$-négligeable de $\mathbb{R}$; ici, $Q_{0}^{u}=[\varphi(u)]^{-1}\{0\}$ avec $\varphi(u) \in H^{1}(Q)$.

Le lemme suivant joue techniquement un rôle fondamental, à la manière du lemme 5 de [7] et s'applique encore à certaines formulations affaiblies.

Lemme 3. - Soit $\xi$ un élément positif de $\mathcal{D}(Q)$; alors pour tout $s \in$ ]$\left.\varphi_{c}, 1\right]$ et toute solution entropique $u$, on dispose de l'estimation d'énergie :

$$
\begin{gathered}
\int_{Q} \operatorname{sign}_{0}(u-s)(\nabla \varphi(u)+(\nu(u)-\nu(s)) \mathbf{G}) \cdot \nabla \xi d x d t-\int_{Q}|u-s| \xi_{t} d x d t \\
=-\lim _{\varepsilon \rightarrow 0^{+}} \frac{1}{\varepsilon} \int_{Q \cap\{-\varepsilon<\varphi(u)-\varphi(s)<\varepsilon\}}|\nabla \varphi(u)|^{2} \xi d x d t .
\end{gathered}
$$

Démonstration. - Puisque $\operatorname{sign}_{\varepsilon}(\varphi(u)-\varphi(s)) \xi$ est un élément de $L^{2}\left(0, T ; H_{0}^{1}(\Omega)\right)$, on a, par le seul fait que $u$ est solution faible, i.e., vérifie l'équation de conservation dans $L^{2}\left(0, T ; H^{-1}(\Omega)\right)$,

$$
\begin{aligned}
& \int_{Q}(\nabla \varphi(u)+(\nu(u)-\nu(s)) \mathbf{G}) \cdot \nabla\left(\operatorname{sign}_{\varepsilon}(\varphi(u)-\varphi(s)) \xi\right) d x d t \\
= & -\int_{0}^{T}\left\langle u_{t}, \operatorname{sign}_{\varepsilon}(\varphi(u)-\varphi(s)) \xi\right\rangle_{H^{-1}(\Omega), H_{0}^{1}(\Omega)} d t \\
= & \int_{Q}\left\{\int_{s}^{u} \operatorname{sign}_{\varepsilon}(\varphi(r)-\varphi(s)) d r\right\} \xi_{t} d x d t .
\end{aligned}
$$

D'où, par passage à la limite, quand $\varepsilon$ tend vers zéro, il vient d'après le lemme 1 :

$$
\begin{gathered}
\int_{Q}|u-s| \xi_{t} d x d t=\int_{Q} \operatorname{sign}_{0}(u-s)(\nabla \varphi(u)+(\nu(u)-\nu(s)) \mathbf{G}) \cdot \nabla \xi d x d t \\
+\lim _{\varepsilon \rightarrow 0^{+}} \int_{Q}(\nabla \varphi(u)+(\nu(u)-\nu(s)) \mathbf{G}) \cdot \nabla\left(\operatorname{sign}_{\varepsilon}(\varphi(u)-\varphi(s))\right) \xi d x d t \\
-171-
\end{gathered}
$$


Suivant la méthode de J. Carrillo [7], on établit que :

$$
\lim _{\varepsilon \rightarrow 0^{+}} I_{\varepsilon}=0, \text { où } I_{\varepsilon}=\int_{Q}(\nu(u)-\nu(s)) \mathbf{G} \cdot \nabla\left(\operatorname{sign}_{\varepsilon}(\varphi(u)-\varphi(s))\right) \xi d x d t \text {. }
$$

En effet, pour $\varepsilon$ suffisamment petit, et d'après le lemme 2, notant $\operatorname{sign}_{\varepsilon}^{\prime}=$ $\frac{1}{\varepsilon} \chi(]-\varepsilon, \varepsilon[):$

$$
I_{\varepsilon}=\int_{Q}\left(\nu \circ\left(\varphi^{-1}\right)_{0}(\varphi(u))-\nu \circ\left(\varphi^{-1}\right)_{0}(\varphi(s))\right)
$$

$$
\operatorname{sign}_{\varepsilon}^{\prime}(\varphi(u)-\varphi(s)) \mathbf{G} \cdot \nabla(\varphi(u)-\varphi(s)) \xi d x d t
$$

où $\left(\varphi^{-1}\right)_{0}$ désigne l'hypo-inverse de $\varphi$, qui est définie par :

$$
\forall r \in[0, \varphi(1)],\left(\varphi^{-1}\right)_{0}(r)=\operatorname{Inf} \varphi^{-1}(\{r\}) .
$$

On pose $H_{\varepsilon}(r)=\int_{0}^{r}\left(\nu \circ\left(\varphi^{-1}\right)_{0}(\tau)-\nu \circ\left(\varphi^{-1}\right)_{0}(\varphi(s))\right) \operatorname{sign}_{\varepsilon}^{\prime}(\tau-\varphi(s)) d \tau$. Alors

$$
I_{\varepsilon}=\int_{Q} H_{\varepsilon}^{\prime}(\varphi(u)) \mathbf{G} \cdot \nabla(\varphi(u)-\varphi(s)) \xi d x d t=-\int_{Q} H_{\varepsilon}(\varphi(u)) \mathbf{G} \cdot \nabla \xi d x d t .
$$

En observant que l'on peut écrire

$$
H_{\varepsilon}(r)=\frac{1}{\varepsilon} \int_{\min (r, \varphi(s)-\varepsilon)}^{\min (r, \varphi(s)+\varepsilon)}\left(\nu \circ\left(\varphi^{-1}\right)_{0}(\tau)-\nu \circ\left(\varphi^{-1}\right)_{0}(\varphi(s))\right) d \tau,
$$

et que $\varphi(s)$ est un point de Lebesgue de $\nu \circ\left(\varphi^{-1}\right)_{0}$, car $\nu \circ\left(\varphi^{-1}\right)_{0}$ est continue en $\varphi(s)$, on en déduit que $\lim _{\varepsilon \rightarrow 0} H_{\varepsilon}(r)=0$.

Par le théorème de convergence dominée de Lebesgue, il s'ensuit que $\lim _{\varepsilon \rightarrow 0} I_{\varepsilon}=0$.

On est alors en mesure d'étendre à la situation du problème $(\mathcal{P})$ un résultat de comparaison classique pour les formulations entropiques des équations hyperboliques non linéaires du premier ordre et qui ouvre la voie à la démonstration de l'unicité. Cela fait l'objet du

THÉORÈME 3 (DE COMPARAISON). - Soient $u_{1}$ et $u_{2}$ deux solutions entropiques associées aux données respectives $u_{0}^{1}$ et $u_{0}^{2}$ vérifiant les hypothèses $\left(\mathcal{H}_{1}\right)$ et $\left(\mathcal{H}_{2}\right)$. Alors, pour tout $\xi \in \mathcal{D}(Q)$, tel que $\xi \geqslant 0$, on a :

$$
\begin{gathered}
\int_{Q} \nabla\left|\varphi\left(u_{1}\right)-\varphi\left(u_{2}\right)\right| \cdot \nabla \xi d x d t \\
+\int_{Q}\left|\nu\left(u_{1}\right)-\nu\left(u_{2}\right)\right| \mathbf{G} \cdot \nabla \xi d x d t-\int_{Q}\left|u_{1}-u_{2}\right| \xi_{t} d x d t \leqslant 0, \\
-172-
\end{gathered}
$$


Formulation forte entropique de lois scalaires hyperboliques-paraboliques dégénérées

i.e., dans $\mathcal{D}^{\prime}(Q)$,

$$
\frac{\partial}{\partial t}\left|u_{1}-u_{2}\right|-\operatorname{div}\left(\left|\nu\left(u_{1}-\nu\left(u_{2}\right) \mid \mathbf{G}\right) \leqslant \Delta\right| \varphi\left(u_{1}\right)-\varphi\left(u_{2}\right) \mid .\right.
$$

Démonstration. - La démonstration est fondée sur la méthode de dédoublement des variables ; on considère donc une fonction $\Phi$ positive, définie sur $Q \times Q$, régulière et vérifiant :

$$
\begin{aligned}
& (s, y) \longmapsto \Phi(t, x, s, y) \in \mathcal{D}(Q) \text { pour tout couple }(t, x) \in Q \\
& (t, x) \longmapsto \Phi(t, x, s, y) \in \mathcal{D}(Q) \text { pour tout couple }(s, y) \in Q .
\end{aligned}
$$

D'après le lemme 1 , on observe au préalable que :

$\operatorname{sign}_{0}\left(u_{1}-u_{2}\right)=\operatorname{sign}_{0}\left(\varphi\left(u_{1}\right)-\varphi\left(u_{2}\right)\right)$ p.p. dans $\left(\left(Q \backslash Q_{0}^{u_{1}}\right) \times Q\right) \cup\left(Q \times\left(Q \backslash Q_{0}^{u_{2}}\right)\right)$.

En prenant $s=u_{2}(t, x)$, et en intégrant sur $Q \backslash Q_{0}^{u_{2}}$ l'égalité du lemme 3, il vient :

$$
\begin{aligned}
& \int_{Q \times\left(Q \backslash Q_{0}^{u_{2}}\right)} \operatorname{sign}_{0}\left(u_{1}-u_{2}\right)\left[\nabla_{y} \varphi\left(u_{1}\right)+\left(\nu\left(u_{1}\right)-\nu\left(u_{2}\right)\right) \mathbf{G}\right] . \nabla_{y} \Phi d x d t d y d s \\
& -\int_{Q \times\left(Q \backslash Q_{0}^{u_{2}}\right)}\left|u_{1}-u_{2}\right| \Phi_{s} d x d t d y d s \\
& \leqslant-\lim _{\varepsilon \rightarrow 0^{+}} \int_{\left(Q \backslash Q_{0}^{u_{1}}\right) \times\left(Q \backslash Q_{0}^{u_{2}}\right)}\left|\nabla_{y} \varphi\left(u_{1}\right)\right|^{2} \operatorname{sign}_{\varepsilon}^{\prime}\left(\varphi\left(u_{1}\right)-\varphi\left(u_{2}\right)\right) \Phi d x d t d y d s,
\end{aligned}
$$

la dernière inégalité étant obtenue par application du lemme 2.

On sait que $u_{1}$ est solution entropique, donc en intégrant sur $Q_{0}^{u_{2}}$ la formulation entropique, on obtient :

$$
\begin{aligned}
& \int_{Q \times Q_{0}^{u_{2}}} \operatorname{sign}_{0}\left(u_{1}-u_{2}\right)\left[\nabla_{y} \varphi\left(u_{1}\right)+\left(\nu\left(u_{1}\right)-\nu\left(u_{2}\right)\right) \mathbf{G}\right] \cdot \nabla_{y} \Phi d x d t d y d s \\
& -\int_{Q \times Q_{0}^{u_{2}}}\left|u_{1}-u_{2}\right| \Phi_{s} d x d t d y d s \leqslant 0 .
\end{aligned}
$$

De même, puisque $u_{2}$ est solution entropique

$$
\begin{aligned}
& \int_{Q \times Q} \operatorname{sign}_{0}\left(u_{1}-u_{2}\right)\left[-\nabla_{x} \varphi\left(u_{2}\right)+\left(\nu\left(u_{1}\right)-\nu\left(u_{2}\right)\right) \mathbf{G}\right] \cdot \nabla_{x} \Phi d x d t d y d s \\
& -\int_{Q \times Q}\left|u_{1}-u_{2}\right| \Phi_{t} d x d t d y d s \\
& \leqslant-\lim _{\varepsilon \rightarrow 0^{+}} \int_{\left(Q \backslash Q_{0}^{u_{1}}\right) \times\left(Q \backslash Q_{0}^{u_{2}}\right)}\left|\nabla_{x} \varphi\left(u_{2}\right)\right|^{2} \operatorname{sign}_{\varepsilon}^{\prime}\left(\varphi\left(u_{1}\right)-\varphi\left(u_{2}\right)\right) \Phi d x d t d y d s .
\end{aligned}
$$




\section{E. Rouvre et G. Gagneux}

En outre, pour presque tout couple $(s, y) \in Q, \operatorname{sign}_{\varepsilon}\left(\varphi\left(u_{1}\right)(s, y)-\varphi\left(u_{2}\right)\right) \Phi$ est un élément de $L^{2}\left(0, T ; H_{0}^{1}(\Omega)\right)$; donc en appliquant une formule de Green, il vient :

$$
\begin{aligned}
& \int_{Q} \nabla_{y} \varphi\left(u_{1}\right) \cdot \nabla_{x}\left(\operatorname{sign}_{\varepsilon}\left(\varphi\left(u_{1}(s, y)\right)-\varphi\left(u_{2}(t, x)\right)\right) \Phi\right) d x d t \\
= & -\int_{Q} \nabla_{x}\left[\nabla_{y} \varphi\left(u_{1}(s, y)\right)\right]\left(\operatorname{sign}_{\varepsilon}\left(\varphi\left(u_{1}(s, y)\right)-\varphi\left(u_{2}(t, x)\right)\right) \Phi d x d t\right. \\
= & 0 .
\end{aligned}
$$

En intégrant sur $Q$ et passant à la limite sur $\varepsilon$, on obtient :

$$
\begin{aligned}
& \int_{Q \times Q} \operatorname{sign}_{0}\left(\varphi\left(u_{1}(s, y)\right)-\varphi\left(u_{2}(t, x)\right)\right) \nabla_{y} \varphi\left(u_{1}\right) \cdot \nabla_{x} \Phi d x d t d y d s \\
&= \lim _{\varepsilon \rightarrow 0^{+}} \int_{Q \times Q} \operatorname{sign}_{\varepsilon}^{\prime}\left(\varphi\left(u_{1}(s, y)\right)-\varphi\left(u_{2}(t, x)\right)\right) \\
& \nabla_{y} \varphi\left(u_{1}\right) \cdot \nabla_{x} \varphi\left(u_{2}\right) \Phi d x d t d y d s .
\end{aligned}
$$

En combinant les résultats des lemmes 1 et 2, le premier membre de l'égalité ci-dessus est égal à :

$$
\int_{Q \times Q} \operatorname{sign}_{0}\left(u_{1}(s, y)-u_{2}(t, x)\right) \nabla_{y} \varphi\left(u_{1}\right) \cdot \nabla_{x} \Phi d x d t d y d s,
$$

et appliquant une dernière fois le lemme 2 , le second se réécrit :

$$
\begin{aligned}
& \lim _{\varepsilon \rightarrow 0^{+}} \int_{\left(Q \backslash Q_{0}^{u_{1}}\right) \times\left(Q \backslash Q_{0}^{u_{2}}\right)} \operatorname{sign}_{\varepsilon}^{\prime}\left(\varphi\left(u_{1}(s, y)\right)\right. \\
&\left.-\varphi\left(u_{2}(t, x)\right)\right) \nabla_{y} \varphi\left(u_{1}\right) \cdot \nabla_{x} \varphi\left(u_{2}\right) \Phi d x d t d y d s .
\end{aligned}
$$

On en déduit finalement l'égalité suivante :

$$
\begin{aligned}
& \lim _{\varepsilon \rightarrow 0^{+}} \int_{\left(Q \backslash Q_{0}^{u_{1}}\right) \times\left(Q \backslash Q_{0}^{u_{2}}\right)} \operatorname{sign}_{\varepsilon}^{\prime}\left(\varphi\left(u_{1}(s, y)\right)\right. \\
\left.-\varphi\left(u_{2}(t, x)\right)\right) \nabla_{y} \varphi\left(u_{1}\right) \cdot \nabla_{x} \varphi\left(u_{2}\right) \Phi d x d t d y d s & \\
= & \int_{Q \times Q} \operatorname{sign}_{0}\left(u_{1}(s, y)-u_{2}(t, x)\right) \nabla_{y} \varphi\left(u_{1}\right) \cdot \nabla_{x} \Phi d x d t d y d s .
\end{aligned}
$$

De manière analogue, on obtient l'égalité :

$$
\begin{aligned}
& -\int_{Q \times Q} \operatorname{sign}_{0}\left(u_{1}-u_{2}\right) \nabla_{x} \varphi\left(u_{2}\right) \cdot \nabla_{y} \Phi d x d t d y d s \\
& =\lim _{\varepsilon \rightarrow 0^{+}} \int_{\left(Q \backslash Q_{0}^{u_{1}}\right) \times\left(Q \backslash Q_{0}^{u_{2}}\right)} \operatorname{sign}_{\varepsilon}^{\prime}\left(\varphi\left(u_{1}\right)-\varphi\left(u_{2}\right)\right) \nabla_{y} \varphi\left(u_{1}\right) \cdot \nabla_{x} \varphi\left(u_{2}\right) \Phi d x d t d y d s .
\end{aligned}
$$


Formulation forte entropique de lois scalaires hyperboliques-paraboliques dégénérées

Il en résulte alors, selon les idées de J.Carrillo [7],

$$
\begin{aligned}
& \int_{Q \times Q} \operatorname{sign}_{0}\left(u_{1}-u_{2}\right)\left(\nabla_{y} \varphi\left(u_{1}\right)-\nabla_{x} \varphi\left(u_{2}\right)\right)\left(\nabla_{y} \Phi+\nabla_{x} \Phi\right) d x d t d y d s \\
+ & \int_{Q \times Q} \operatorname{sign}_{0}\left(u_{1}-u_{2}\right)\left(\nu\left(u_{1}\right)-\nu\left(u_{2}\right)\right) \mathbf{G} \cdot\left(\nabla_{y} \Phi+\nabla_{x} \Phi\right) d x d t d y d s \\
- & \int_{Q \times Q}\left|u_{1}-u_{2}\right|\left(\Phi_{t}+\Phi_{s}\right) d x d t d y d s \\
\leqslant & -\lim _{\varepsilon \rightarrow 0^{+}} \int_{\left(Q \backslash Q_{0}^{u_{1}}\right) \times\left(Q \backslash Q_{0}^{u_{2}}\right)} \operatorname{sign}_{\varepsilon}^{\prime}\left(\varphi\left(u_{1}\right)-\varphi\left(u_{2}\right)\right) \Phi \mid \nabla_{y} \varphi\left(u_{1}\right) \\
\leqslant & 0 .
\end{aligned}
$$

Alors, pour $\xi \in \mathcal{D}(Q)$ tel que $\xi \geqslant 0$, on considère la fonction

$$
\Phi(t, x, s, y)=\xi\left(\frac{t+s}{2}, \frac{x+y}{2}\right) \rho_{l}\left(\frac{t-s}{2}\right) \rho_{n}\left(\frac{x-y}{2}\right)
$$

où $\rho_{l}$ et $\rho_{n}$ sont des suites régularisantes. Ainsi,

$$
\begin{aligned}
& \int_{Q \times Q} \operatorname{sign}_{0}\left(u_{1}-u_{2}\right)\left(\nabla_{y} \varphi\left(u_{1}\right)-\nabla_{x} \varphi\left(u_{2}\right)\right) \cdot \nabla \xi\left(\frac{t+s}{2}, \frac{x+y}{2}\right) \rho_{l} \rho_{n} d x d t d y d s \\
& +\int_{Q \times Q}\left|\nu\left(u_{1}\right)-\nu\left(u_{2}\right)\right| \mathbf{G} \cdot \nabla \xi\left(\frac{t+s}{2}, \frac{x+y}{2}\right) \rho_{l} \rho_{n} d x d t d y d s \\
& -\int_{Q \times Q}\left|u_{1}-u_{2}\right| \frac{\partial \xi}{\partial t}\left(\frac{t+s}{2}, \frac{x+y}{2}\right) \rho_{l} \rho_{n} d x d t d y d s \leqslant 0 .
\end{aligned}
$$

On conclut en faisant tendre $l$ et $n$ vers l'infini, selon l'idée fondatrice de S.N. Kruzhkov, par la procédure classique du traitement des problèmes hyperboliques du premier ordre non linéaires (cf. [1], [11], [19] par exemple), étendue au cas d'un terme de diffusion par J. Carrillo (cf. [6], [7] et sa bibliographie ou [10]) et en utilisant la définition des points de Lebesgue.

\section{Unicité lorsque $\Omega=] 0,1[$}

Pour établir un résultat d'unicité, on tire profit de simplifications liées à la dimension 1 , en introduisant l'espace $B V_{x}(Q)$ selon les travaux de Wu Zhuoqun et Yin Jingxue [23]. Cependant, on utilisera une méthode différente de celle mise en œuvre par ces auteurs qui s'appuient essentiellement sur la connaissance de l'onde de choc et des propriétés des fonctions à variation bornée, pour établir dans ce cadre un formulaire de type Gauss-Green. 
Remarque 1. - Par le choix de la dimension 1 en espace, $\frac{\partial \varphi(u)}{\partial x}$ est un élément de $B V_{x}(Q)=\left\{f \in L_{l o c}^{1}(Q), \frac{\partial f}{\partial x} \in \mathcal{M}_{b}(Q), Q=\right] 0, T[\times] 0,1[\}$. Ceci permet de donner un sens à la dérivée normale de $\varphi(u)$ le long de $\Sigma$; en effet (cf. lemme 2.2, p.318 de [23]), pour presque tout $t \in] 0, T\left[,\left(\frac{\partial \varphi(u)}{\partial x}\right)(t,.) \in\right.$ $\overline{B V}(] 0,1[)$, et donc, au sens des traces dans $\overline{B V}(] 0,1[)$, on peut introduire $\gamma\left(\frac{\partial \varphi(u)}{\partial x}\right)(t, .)_{\left.\right|_{x=0}}$ et $\gamma\left(\frac{\partial \varphi(u)}{\partial x}\right)(t, .)_{\left.\right|_{x=1}}$ i.e., par commodité de notation, $\frac{\partial \varphi(u)}{\partial x}(t, 0)$ et $\frac{\partial \varphi(u)}{\partial x}(t, 1)$, et donc pour presque tout $\left.t \in\right] 0, T\left[\frac{\partial \varphi(u)}{\partial n}(t,\right.$.$) .$ La condition de bord (1) est alors écrite de manière rigoureuse grâce à une formule de Gauss-Green licite ; nous parlerons alors de solutions fortes entropiques.

Par ailleurs, en dimension 1, le résultat d'existence est enrichi par le fait que toute solution entropique $u$ est telle que $\varphi(u) \in \mathcal{C}^{0}(\bar{Q})$ :

en effet, d'après le lemme 8.1 p.297 de J.L. Lions et E. Magenes [12], le représentant de $\varphi(u)$ peut être choisi scalairement continu de $[0, T]$ dans $H_{0}^{1}(\Omega)$, indépendamment de la dimension de l'espace ; or, en dimension 1 , on sait qu'alors, pour tout $t$ de $[0, T]$, la fonction $x \longmapsto(\varphi(u))(t, x)$ est höldérienne d'ordre $1 / 2$, uniformément par rapport à $t$, ce qui, joint au fait que $\varphi(u)$ est en particulier continue de $[0, T]$ dans $L^{1}(] 0,1[)$, implique par application du lemme d'Ascoli que $\varphi(u) \in \mathcal{C}^{0}([0, T] \times[0,1])$. En conséquence, $\left\{(t, x) \in \bar{Q}, 0 \leqslant u(t, x) \leqslant \varphi_{c}\right\}$ (zone d'hyperbolicité) est un fermé et $\{(t, x) \in Q, u(t, x)>0\}$ (zone de parabolicité) est un ouvert.

De plus, en dimension 1 , on dispose du procédé de régularisation suivant :

Proposition 1.- Soit $X=\left\{U \in L^{2}\left(0, T ; H_{0}^{1}(\Omega)\right) \cap H^{1}(Q), U \geqslant\right.$ $0 \quad \mathcal{L}^{2}-$ p.p. dans $\left.Q, \Delta U \in \mathcal{M}_{b}(Q)\right\}$. Alors, pour tout $U \in X$, on peut construire une suite généralisée régularisée $\left(U_{\varepsilon}\right)_{\varepsilon>0} \subset X \cap \mathcal{C}^{\infty}(\bar{Q})$ convergeant vers $U$ dans $H^{1}(Q)$, telle que :

$$
\forall \Phi \in \mathcal{C}^{0}(\bar{Q}), \lim _{\varepsilon \rightarrow 0^{+}} \int_{Q}\left(\Delta U_{\varepsilon}\right) \Phi d x d t=\int_{Q} \Phi d[\Delta U] .
$$

Principe de la démonstration. - On construit un ouvert $\tilde{Q}$ tel que $\bar{Q} \varsubsetneqq$ $\tilde{Q}$, de telle sorte que $\tilde{U}$ soit le prolongement par réflexion de $U$ sur $\tilde{Q}$. Ainsi, on ne crée aucun saut sur la fonction $\tilde{U}$ et sur sa dérivée normale le long de $\Sigma$. Si $\left(\eta_{\varepsilon}\right)_{\varepsilon>0}$ est une suite régularisante, du fait de la construction, on montre que $U_{\varepsilon}=\left(\tilde{U} * \eta_{\varepsilon}\right)_{\left.\right|_{Q}}$ vérifie les propriétés désirées. En particulier, on 
montre que $U_{\varepsilon}$ est positif en utilisant le fait que pour tout $\varepsilon>0, \eta_{\varepsilon}$ est une fonction paire et décroissante sur $\mathbb{R}^{+}$.

Conséquence 1. - Le procédé d'approximation décrit à la proposition 1 permet d'établir que si $U$ est un élément de $\mathrm{X}$, alors

$$
\begin{gathered}
\left.\frac{\partial U}{\partial n} \leqslant 0 \text { le long de } \Sigma \text { au sens où p.p. en } t \in\right] 0, T\left[, \frac{\partial U}{\partial x}(t, 1) \leqslant 0,\right. \\
-\frac{\partial U}{\partial x}(t, 0) \leqslant 0 .
\end{gathered}
$$

On en déduit alors que

$$
\frac{\partial \varphi(u)}{\partial n} \leqslant 0 \quad \mathcal{H}^{1}-\text { p.p. sur } \Sigma .
$$

\subsection{Interprétation de la condition de bord et de choc}

Soit $\mathbf{n}$ le vecteur normal unitaire sortant à la frontière $\Sigma$.

On définit $\Sigma^{+}=\{(t, x) \in \Sigma$, G.n $>0\}$. Pour fixer les idées, on prend $\left.\Sigma^{+}=\right] 0, T[\times\{1\}$ et $\mathbf{G}=g \mathbf{i}$, avec $g>0$.

En considérant différentes valeurs de $s$ comprises entre 0 et 1 , la formulation entropique impose des conditions sur la solution $u$ le long de $\Sigma$ et de l'onde de choc $\Gamma_{u}$, complémentaire de l'ensemble des points de $\mathcal{L}^{2}$-approximative continuité de $u$, dont la normale unitaire est notée $v=$ $\left(v_{t}, v_{x}\right)$.

Selon les notations de Wu Zhuoqun et Yin Jingxue [23] et introduisant $f^{g}$ et $f^{d}$ les limites à gauche et à droite d'une fonction numérique $f$ à variation bornée et $\widetilde{f}$ leur moyenne arithmétique, on a

PropriÉtÉ 1. - Toute solution entropique de $(\mathcal{P})$ vérifie, $\mathcal{H}^{1}-p . p$. sur $\Gamma_{u}$, la condition de Rankine-Hugoniot :

$$
\left\{\begin{array}{c}
\left(u^{+}-u^{-}\right) v_{t}=\left(\nu\left(u^{+}\right)-\nu\left(u^{-}\right) g v_{x}+\left[\left(\widehat{\varphi^{\prime}}(u) \frac{\partial u}{\partial x}\right)^{d}-\left(\widehat{\varphi^{\prime}}(u) \frac{\partial u}{\partial x}\right)^{g}\right]\left|v_{x}\right|,\right. \\
0 \leqslant u^{-} \leqslant \varphi_{c}, 0 \leqslant \varphi_{c}^{+},
\end{array}\right.
$$

et l'inégalité de saut : pour tout $s \in\left[0, \varphi_{c}\right]$,

$$
\begin{gathered}
{\left[\operatorname{sign}_{0}\left(u^{+}-s\right)-\operatorname{sign}_{0}\left(u^{-}-s\right)\right]} \\
\qquad(\bar{u}-s) v_{t}-(\overline{\nu(u)}-\nu(s)) g v_{x}-\left(\widehat{\left.\varphi^{\prime}(u) \frac{\partial u}{\partial x}\right)} v_{x}\right] \leqslant 0, \\
-177-
\end{gathered}
$$


où :

$u^{+}\left(t_{0}, x_{0}\right)$ et $u^{-}\left(t_{0}, x_{0}\right)$ représentent les limites approximatives de $u$ en un point $\left(t_{0}, x_{0}\right)$ de $\Gamma_{u}, \bar{u}\left(t_{0}, x_{0}\right)$ désigne leur moyenne arithmétique, et $\widehat{\varphi^{\prime}}$ est un représentant borélien borné de la classe de $\varphi^{\prime}$ tel que, par la propriété de superposition fonctionnelle, on ait $\frac{\partial \varphi(u)}{\partial x}=\widehat{\varphi^{\prime}}(u) \frac{\partial u}{\partial x}$.

Remarque 2. - Pour établir l'unicité de la solution entropique, divers auteurs ont utilisé un résultat de A.I. Vol'pert et S.I. Hudjaev [21] qui s'avère incorrect ( $c f$. sur ce point [22], [23]). En effet, dans l'inégalité des sauts, ces auteurs ont omis le terme $\left(\widehat{\varphi^{\prime}}(u) \frac{\partial u}{\partial x}\right)$, qui en fait contribue à l'expression des sauts. Comme l'exposent $\mathrm{Ph}$. Bénilan et $\mathrm{F}$. Bouhsiss dans leur contre-exemple [2], il est difficile de dissocier le terme hyperbolique et le terme parabolique.

Proposition 2. - Toute solution forte entropique u du problème $(\mathcal{P})$ vérifie les conditions de bord:

$$
\left\{\begin{array}{l}
0 \leqslant \gamma u \leqslant \varphi_{c} \quad \mathcal{H}^{1}-p . p . \text { sur } \Sigma \\
\nu(\gamma u(t, 1)) g+\frac{\partial \varphi(u)}{\partial x}(t, 1) \leqslant 0 \quad \mathcal{H}^{1}-p . p . \text { sur } \Sigma^{+}
\end{array}\right.
$$

Démonstration. - 1) Puisque $\varphi$ est, en particulier, une fonction lipschitzienne sur $[0,1]$, grâce à la propriété de superposition fonctionnelle sur $\overline{B V}(Q)$, on a $\gamma(\varphi(u))=\varphi(\gamma(u))$. Or $\varphi(u)_{\left.\right|_{\Sigma}}=0$ et on en déduit donc que $0 \leqslant \gamma u \leqslant \varphi_{c} \mathcal{H}^{1}-p . p$. sur $\Sigma$. Cependant, ce résultat n'est pas propre à la dimension 1 , on a plus généralement :

$$
0 \leqslant \gamma u \leqslant \varphi_{c} \mathcal{H}^{n}-\text { p.p. sur } \Sigma .
$$

2) Dans la formulation entropique sur le bord (3.1), désormais écrite de façon rigoureuse, on prend $s=0$; ainsi

$$
\operatorname{sign}_{0}(\gamma u) \nu(\gamma u) \text { G.n } \leqslant-\frac{\partial \varphi(u)}{\partial n} \quad \mathcal{H}^{1}-p . p . \operatorname{sur} \Sigma .
$$

Or $\nu$ est croissante, nulle en 0 et donc $\operatorname{sign}_{0}(\gamma u) \nu(\gamma u)=\nu(\gamma u) \geqslant 0$. L'inégalité (5.2) est par conséquent toujours vérifiée sur $\Sigma \backslash \Sigma^{+}$.

Il vient alors pour presque tout $t \in] 0, T[$, la condition

$$
\nu(\gamma u(t, 1)) g \leqslant-\frac{\partial \varphi(u)}{\partial x}(t, 1) \mathcal{H}^{1}-p . p . \operatorname{sur} \Sigma^{+}
$$


Formulation forte entropique de lois scalaires hyperboliques-paraboliques dégénérées

On peut observer que lorsque $\varphi$ est identiquement nulle (i.e. ici, lorsque $\varphi_{c}=1$ ) ou lorsque les effets de diffusion sont inexistants (i.e. par exemple, lorsque $0 \leqslant u_{0} \leqslant \varphi_{c}<1$ ), la proposition 2 se réduit à la condition classique: $\nu(\gamma u(t, 1))=0, \mathcal{H}^{1}-p . p$. sur $\Sigma^{+}(c f .[1],[10]$, p.169 ou [19], vol. II, p.271). Dans le cas général, les effets de la diffusion et du transport convectif sur le bord $\Sigma^{+}$sont liés par la relation (5.3), dont l'interprétation sera donnée dans la preuve du lemme 5 et à la remarque 3 .

\subsection{Résultat d'unicité lorsque $\Omega=] 0,1[$}

Dans cette partie, $u_{1}$ et $u_{2}$ désignent des solutions fortes entropiques associées aux données respectives $u_{0}^{1}$ et $u_{0}^{2}$, vérifiant les hypothèses $\left(\mathcal{H}_{1}\right)$ et $\left(\mathcal{H}_{2}\right)$. On établit alors deux résultats préparatoires.

LeMme 4. - Soit $\Phi \in \mathcal{D}(] 0, T[)$, tel que $\Phi \geqslant 0$; alors

$$
\begin{aligned}
\int_{Q}\left|u_{1}-u_{2}\right| \Phi^{\prime}(t) d x d t+ & \left\langle\left\langle\frac{\partial\left|\varphi\left(u_{1}\right)-\varphi\left(u_{2}\right)\right|}{\partial n}, \Phi \otimes 1_{\Omega}\right\rangle\right\rangle \\
& +\int_{\Sigma}\left|\nu\left(u_{1}\right)-\nu\left(u_{2}\right)\right| \text { G.n } d \sigma d t \geqslant 0 .
\end{aligned}
$$

Démonstration. - Considérons, dans le théorème 3 de comparaison, la suite de fonctions test $\left(\xi_{j}\right)_{j}$ définie par $\xi_{j}(t, x)=\Phi(t)\left(1-\rho_{j}(x)\right) \in H_{0}^{1}(Q)$, où $\left(\rho_{j}\right)_{j}$ est une suite $\operatorname{de} \mathcal{C}^{\infty}(\bar{\Omega})$ telle que :

$$
\forall x \in \bar{\Omega}, 0 \leqslant \rho_{j}(x) \leqslant 1,\left(\rho_{j}\right)_{\left.\right|_{\Sigma}}=1, \rho_{j} \longrightarrow 0 \text { partout dans } \Omega ;
$$

ce choix est loisible par densité de $\mathcal{D}(Q)$ dans $H_{0}^{1}(Q)$. On obtient donc :

$$
\begin{aligned}
& \int_{Q} \nabla\left|\varphi\left(u_{1}\right)-\varphi\left(u_{2}\right)\right| \cdot \nabla \rho_{j}(x) \Phi(t) d x d t+\int_{Q}\left|\nu\left(u_{1}\right)-\nu\left(u_{2}\right)\right| \mathbf{G} \cdot \nabla \rho_{j}(x) \Phi(t) d x d t \\
& +\int_{Q}\left|u_{1}-u_{2}\right| \Phi^{\prime}(t)\left(1-\rho_{j}(x)\right) d x d t \geqslant 0 .
\end{aligned}
$$

Or, selon la définition de la dérivée normale donnée à la définition 1 et du fait que $\left|\varphi\left(u_{1}\right)-\varphi\left(u_{2}\right)\right| \in H^{1}(Q) \cap L^{2}\left(0, T ; H_{0}^{1}(\Omega)\right)$ avec $\Delta\left|\varphi\left(u_{1}\right)-\varphi\left(u_{2}\right)\right| \epsilon$ $\mathcal{M}_{b}(Q)$, on a :

$$
\begin{aligned}
\int_{Q} \nabla\left|\varphi\left(u_{1}\right)-\varphi\left(u_{2}\right)\right| . \nabla \rho_{j}(x) \Phi(t) d x d t & =\left\langle\left\langle\frac{\partial\left|\varphi\left(u_{1}\right)-\varphi\left(u_{2}\right)\right|}{\partial n}, \Phi \rho_{j}\right\rangle\right\rangle \\
& -\int_{Q} \rho_{j}(x) \Phi(t) d\left[\Delta\left|\varphi\left(u_{1}\right)-\varphi\left(u_{2}\right)\right|\right]
\end{aligned}
$$


On sait que la suite uniformément bornée de fonctions continues $\Phi \rho_{j}$ converge simplement, partout dans $Q$, vers la fonction nulle.

Ainsi, en appliquant à cette suite le théorème de convergence dominée pour la mesure de Borel sommable sur $Q, \Delta\left|\varphi\left(u_{1}\right)-\varphi\left(u_{2}\right)\right|$, il vient

$\lim _{j \rightarrow+\infty} \int_{Q} \nabla\left|\varphi\left(u_{1}\right)-\varphi\left(u_{2}\right)\right| . \nabla \rho_{j}(x) \Phi(t) d x d t=\left\langle\left\langle\frac{\partial\left|\varphi\left(u_{1}\right)-\varphi\left(u_{2}\right)\right|}{\partial n}, \Phi \otimes 1_{\Omega}\right\rangle\right\rangle$.

De manière analogue, après une intégration de type Gauss-Green généralisée dans $L^{1}(Q) \cap \overline{B V}(Q)$,

$\lim _{j \rightarrow+\infty} \int_{Q}\left|\nu\left(u_{1}\right)-\nu\left(u_{2}\right)\right| \mathbf{G} . \nabla \rho_{j}(x) \Phi(t) d x d t=\int_{\Sigma}\left|\nu\left(\gamma u_{1}\right)-\nu\left(\gamma u_{2}\right)\right| \mathbf{G} . \mathbf{n} \Phi d \sigma d t$.

En faisant tendre $j$ vers l'infini, on obtient le résultat voulu.

LEMME 5. - Lorsque $\Omega=] 0,1[$, on dispose de la relation

$$
\frac{\partial\left|\varphi\left(u_{1}\right)-\varphi\left(u_{2}\right)\right|}{\partial n}+\left|\nu\left(\gamma u_{1}\right)-\nu\left(\gamma u_{2}\right)\right| \text { G.n } \leqslant 0 \quad \text { p.p. sur } \Sigma \text {. }
$$

Démonstration. - On peut sans restriction supposer que $\nu$, fonction croissante, nulle en 0 , est telle que $\nu\left(\varphi_{c}\right) \neq 0\left(\operatorname{sinon} \nu \equiv 0\right.$ sur $\left[0, \varphi_{c}\right]$ et l'inégalité recherchée est toujours vérifiée).

D'après la condition d'entropie sur la frontière, on a deux possibilités :

i) ou bien, $\nu(\gamma u(t, 1))=0$ et $\gamma\left(\frac{\partial \varphi(u)}{\partial x}(t, 1)\right)=0$. En effet, si $\nu(\gamma u(t, 1))=$ 0 , alors $\gamma u(t, 1)<\varphi_{c}$, ce qui implique en dimension 1 que $\gamma\left(\frac{\partial \varphi(u)}{\partial x}(t, 1)\right)=$ 0 ;

ii) ou bien $\nu(\gamma u(t, 1))>0$ et alors, d'après la formulation sur le bord énoncée par la proposition $2, \gamma\left(\frac{\partial \varphi(u)}{\partial x}(t, 1)\right)<0\left(\right.$ i.e. $\left.\gamma u(t, 1)=\varphi_{c}\right)$.

On distingue deux cas pour tenir compte de cette alternative sur $\Sigma^{+}$:

$1^{\mathrm{er}}$ cas $: \nu\left(\gamma u_{1}\right)=\nu\left(\gamma u_{2}\right)$, alors $\operatorname{sign}_{0}\left(\gamma u_{1}-\gamma u_{2}\right)\left(\nu\left(\gamma u_{1}\right)-\nu\left(\gamma u_{2}\right)\right)=0$. L'assertion (5.4) s'écrit donc :

$$
\frac{\partial\left|\varphi\left(u_{1}\right)-\varphi\left(u_{2}\right)\right|}{\partial n} \leqslant 0
$$

ce qui est toujours le cas d'après la conséquence 1. 
Formulation forte entropique de lois scalaires hyperboliques-paraboliques dégénérées

$2^{\text {ème }}$ cas : $\gamma u_{1}=\varphi_{c}$ et $\nu\left(\gamma u_{2}\right)=0$ (pour fixer les idées ; le cas $\gamma u_{2}=\varphi_{c}$ et $\nu\left(\gamma u_{1}\right)=0$ se traitant de manière similaire).

Sur $\Sigma^{+}$, on observe que $\operatorname{sign}_{0}\left(\gamma u_{1}-\gamma u_{2}\right)\left(\nu\left(\gamma u_{1}\right)-\nu\left(\gamma u_{2}\right)\right)=\nu\left(\gamma u_{1}\right)$ et $\frac{\partial\left|\varphi\left(u_{1}\right)-\varphi\left(u_{2}\right)\right|}{\partial n}=\frac{\partial \varphi\left(u_{1}\right)}{\partial n}$; l'inégalité (5.4) s'écrit alors :

$$
\nu\left(\gamma u_{1}(t, 1)\right) g+\frac{\partial \varphi\left(u_{1}\right)}{\partial x}(t, 1) \leqslant 0,
$$

ce qui est toujours vrai d'après l'interprétation de la condition de bord ( $c f$. proposition 2).

Sur $\Sigma \backslash \Sigma^{+}$, puisque $\operatorname{sign}_{0}\left(\gamma u_{1}-\gamma u_{2}\right)\left(\nu\left(\gamma u_{1}\right)-\nu\left(\gamma u_{2}\right)\right)$ G.n $\leqslant 0$ et $\frac{\partial\left|\varphi\left(u_{1}\right)-\varphi\left(u_{2}\right)\right|}{\partial n} \leqslant 0$, on a directement le résultat recherché.

Remarque 3. - Dans le cadre considéré ici, i.e. lorsque la fonction $\nu$ est croissante au sens large, avec $\nu(0)=0$ et $\nu\left(\varphi_{c}\right)>0, \varphi_{c}>0$, la démonstration du lemme 5 met en lumière le fait que toute solution forte entropique $u$ est soumise à une contrainte unilatérale sur $\Sigma^{+}$au sens de l'alternative suivante :

$$
\begin{gathered}
\text { ou bien }\left\{\begin{array}{l}
0 \leqslant(\gamma u)<\varphi_{c} \\
\nu(\gamma u)=0
\end{array} \text { et } \frac{\partial \varphi(u)}{\partial n}=0\right. \\
\text { ou bien } \gamma \mathrm{u}=\varphi_{c} \text { et } \frac{\partial \varphi(u)}{\partial n} \leqslant-g \nu\left(\varphi_{c}\right)<0,
\end{gathered}
$$

i.e., sur $\Sigma^{+}$, le flux diffusif est nul lorsque la saturation $u$ n'y atteint pas la valeur critique $\varphi_{c}$. Les inégalités d'entropie introduites à la définition 2 induisent une formulation en termes d'inéquations variationnelles, qui, outre la condition de saut, traduisent un problème unilatéral sur $\Sigma^{+}$. Lorsque la fonction $\nu$ est strictement croissante, la trace de $u$ sur $\Sigma^{+}$est soit nulle, soit égale à $\varphi_{c}$.

On est alors en mesure d'énoncer le résultat d'unicité et de stabilité.

THÉORÈME 4. - Soient $u_{1}$ et $u_{2}$ deux solutions fortes entropiques associées aux données initiales respectives $u_{0}^{1}$ et $u_{0}^{2}$ vérifiant les hypothèses $\left(\mathcal{H}_{1}\right)$ et $\left(\mathcal{H}_{2}\right)$. Alors

$$
\forall t \in[0, T],\left|u_{1}(t)-u_{2}(t)\right|_{L^{1}(\Omega)} \leqslant\left|u_{0}^{1}-u_{0}^{2}\right|_{L^{1}(\Omega)} ;
$$

plus précisément, on a

$$
\forall t \in[0, T], \int_{\Omega}\left(u_{1}(t, x)-u_{2}(t, x)\right)^{+} d x \leqslant \int_{\Omega}\left(u_{0}^{1}(x)-u_{0}^{2}(x)\right)^{+} d x .
$$


Démonstration. - Soit $\Phi \in \mathcal{D}(] 0, T[), \Phi \geqslant 0$; en introduisant la fonction numérique continue sur $[0, T]$, définie pour tout $t$ de $[0, T]$ par $\Psi(t)=$ $\int_{\Omega}\left|u_{1}(t, x)-u_{2}(t, x)\right| d x$, on a, d'après les lemmes 4 et 5 :

$$
\int_{0}^{T} \Psi(t) \Phi^{\prime}(t) d t \geqslant 0
$$

donc $\frac{d \Psi}{d t}$ est une distribution négative de $\mathcal{D}^{\prime}([0, T[)$, i.e. selon [18], p.29 et $53-54, \Psi$ est une fonction continue décroissante sur $[0, T]$, d'où la propriété recherchée, qui entraîne l'unicité.

La seconde inégalité établit l'existence d'un semi-groupe fortement continu de T-contraction opérant dans $L^{1}(\Omega)$, via l'application $u_{0} \longmapsto S(t) u_{0}=$ $u(t,),. t>0$ et dégage un principe de comparaison et de conservation de l'ordre ; elle résulte immédiatement par hérédité du fait que cette propriété est vraie pour les solutions $u_{\varepsilon}$ approchées par viscosité et de la propriété d'unicité de la solution entropique $u$, point d'accumulation dans $\mathcal{C}^{0}\left([0, T] ; L^{1}(\Omega)\right)$ de la suite $\left(u_{\varepsilon}\right)_{\varepsilon>0}$.

Lorsque $\Omega$ est un ouvert de frontière de classe $W^{2,+\infty}$, le résultat d'unicité peut être étendu au cas multidimensionnel. Sous cette condition, on montre que le flux $\frac{\partial \varphi(u)}{\partial n}$ est un élément de $L^{\infty}\left(0, T ; L^{1}(\Gamma)\right)$ permettant ainsi de définir la condition entropique frontalière en termes d'intégrale de Lebesgue sur le bord et d'en donner une interprétation rigoureuse ; ce point de vue est développé par E. Rouvre et G. Gagneux dans [17].

\section{Bibliographie}

[1] BARdos (C.), LERouX (A.Y.) et NÉdÉLEC (J.C.). - First order quasilinear equations with boundary conditions, Comm. in partial differential equations, 4(9), (1979), pp. 1017-1034.

[2] BÉnilan (Рн.) et Bouhsiss (F.). - Un contre-exemple dans le cadre des équations hyperboliques paraboliques dégénérées, Publication Mathématique de Besançon, Analyse non linéaire 15, (1995/97), pp. 123-126.

[3] BÉNILAN (РH.), GARIEPY (R.F.). - Strong solutions in $L^{1}$ of degenerate parabolic equations, J. of differential equations 119, (1995), pp. 473-502.

[4] BÉNILAN (Рн.) et TouRÉ (H.). - Sur l'équation générale $u_{t}=a\left(., \varphi(., u)_{x}\right)_{x}+v$ dans $L^{1}$ : II le problème d'évolution, Annal. Inst. H. Poincaré, vol. 12, $\mathrm{n}^{0} 6,(1995)$, pp. 727-761.

[5] BÜRGER (R.) et WENDLAND (W.L.). - Existence, uniqueness and stability of generalized solutions of an initial-boundary value problem for a degenerating quasilinear 
Formulation forte entropique de lois scalaires hyperboliques-paraboliques dégénérées

parabolic equation, J. of Mathematics, Analysis and applications 218, (1998), pp. 207-239.

[6] CARrillo (J.). - Solutions entropiques de problèmes non linéaires dégénérés, C.R. Acad. Sci. Paris, t. 327, série I, (1998), pp. 155-160.

[7] CARRILlo (J.). - Entropy solutions for nonlinear degenerate problems, Archi. Rational Mech. Anal., vol. 147, $\mathrm{n}^{0} 4$, (1999), pp. 269-361.

[8] DaUtray (R.) et Lions (J.L.). - Analyse mathématique et calcul numérique pour les sciences et les techniques, Collection enseignement, Masson, vol. 2, (1987).

[9] DiAZ (J.I.) et VRABIE (I.I.). - Compactness of the Green operator of nonlinear diffusion equations: Application to Boussinesq type systems in fluid dynamics, J. of Juliusz Schauder Center, vol. 4, (1994), pp. 399-416.

[10] GaGneuX (G.) et MADAune-TORT (M.). - Analyse mathématique de modèles non linéaires de l'ingénierie pétrolière, Collection Mathématiques et Applications, Springer-Verlag, vol. 22, (1996).

[11] GodlewSKI (E.) et RAVIART (P. A.). - Hyperbolic systems of conservation laws, Mathématiques \& Applications, Ed. Ellipses, SMAI, vol. 3/4, (1991).

[12] Lions (J.L.) et MAGenes (E.). - Problèmes aux limites non homogènes et applications, Dunod, vol. 1, (1968).

[13] Magenes (E.). - Sur l'operateur du type Dirichlet-Neumann pour certaines équations paraboliques non linéaires, Eq. aux dérivées partielles et applications, Articles dédiés à J.L.Lions, Gauthiers-Villars, (1998), pp. 655-665.

[14] MALIKI (M.) et TouRÉ (H.).- Solution généralisée locale d'une équation parabolique quasilinéaire dégénérée au second ordre, Prépublication de l'équipe de mathématiques de Besançon, Université de Franche-Comté, (1995), pp. 1-16.

[15] Marcus (M.) et Mizel (V.J.). - Absolute continuity on Tracks and Mappings of Sobolev Spaces, Archi. Rational Mech. Anal., vol. 45, no 4, (1972), pp. 294-320.

[16] Отто (F.). - Initial-boundary value problem for a scalar conservation law, C.R. Acad. Sci. Paris, t. 322, série I, (1996), pp. 729-734.

[17] Rouvre (E.) et GaGneuX (G.). - Solution forte entropique de lois scalaires hyperboliques-paraboliques dégénérées, C.R. Acad. Sci. Paris, t.329, série I, (1999), pp. 599-602.

[18] Schwartz (L.). - Théorie des distributions, Hermann,(1978).

[19] SERRE (D.). - Systèmes de lois de conservation I et II, Fondations, Diderot Editeur, Arts et sciences, (1996).

[20] URRUTY (P. ). - Sur une équation autonome d'évolution doublement non linéaire et dégénérée, C.R. Acad. Sci. Paris, t. 322, série I, (1996), pp. 741-744.

[21] VOL'PERT (A.I.). - HUDJAEV (S.I.).- Cauchy's problem for degenerate second order quasilinear parabolic equation, Math Sbornik V. 78(120), $\mathrm{n}^{0} 3$, (1969).

[22] WU ZHUOQUN. - A free boundary problem for degenerate quasilinear parabolic equations, Nonlinear Analysis, vol. 9, $\mathrm{n}^{0}$ 9, (1985), p. 937-951.

[23] WU Zhuoqun et YIN JingXue. - Some properties of functions in $B V_{x}$ and their applications of the uniqueness of solutions for degenerate quasilinear parabolic equations, Northeastern Math. J., 5(4), (1989), p. 395-422. 